'timiaturus, turibulum', mhd. kclle, hundcslkclle, huntchılle 'hundehïtte', auch 'verächtliches gefängnis für menschen'. Weitere beispiele unseres wortes mit dem bedeutungsiibergang 'gefäß' : 'haus' (woriiber vgl. Persson, Beitr. \%. indogerm. wortforschung öfters, z. b. s. 100 ff.) liefern die ahd. formen wîtkelli 'porticus', furikclli 'vestibulum, camera, proscenia, vomitoria'. Über den wechsel leella f. : kelli n. vgl. got. ludja f. : ahd. ant-lutti n., ahd. fenna f. : got. fani n., ahd. rippa f. : rippi n., ahd. kliuwa f. : lsliuwi n. u.a.

LUND. N. OTTO HEINERTZ.

\title{
SPRECHEN MIT DEM ACCUSATIV DER PERSON.
}

Im 40. band dieser zeitschrift s. 412 ff. hat Erik Wellander eine neue erklärung für den auffälligen accusativ der person beim verbum sprechen vorgeschlagen. Er verwirft die annahme einer objectsvertauschung oder einer nachwirkung 'des noch nicht völlig erloschenen gefühls für die ganz allgemeine bedeutung des accusativs' und meint, indem er an einen beleg im ahd. Tatian anknüpft, in der construction einen fall von 'verschiebung der syntaktischen gliederung' erblicken zu dürfen. Obgleich man nun in dem negativen teil dieses ergebnisses dem schwedischen gelehrten beistimmen kann, so erregt der positive teil manclie bedenken, welche sich nicht gegen die annahme einer solchen verschiebung überhaupt richten (es gibt deren ja unzweifelhafte beispiele), sondern in dem tatsächlich vorliegenden material begrïndet sind.

Vor allem ist festzustellen daB sprcchen mit dem accusativ der person keineswegs auf das hochdeutsche beschränkt ist. Wellander selbst führt einen mittelniederdentschen beleg an, wenn er ihn auch nur zögernd gelten lassen will ('Vielleicht. berult die construction hier auf hd. einfluB' a. a. o. s. 412), aber möglicherweise gehört anch eine zweite, an sich nicht. entscheidende stelle aus dem nämlichen mnd. fext, hierher (oli sprack ite $B$. Körner 75 b, s. Schiller-Jübben), und anBerde'll 
läßt sich die construction aus dem mittelniederländischen, dem altenglischen und wohl auch dem friesischen nachweisen, wie sich weiter unten ergeben wird.

Fassen wir aber zunächst die von Wellander versuchte erklairung ins auge. Von dem gedanken ausgehend daß die seiner meinung nach 'nicht aus der gewöhnlichen anwendung des verbums (sprechen) heraus' erklärliche transitivität 'irgendwie aus einem anderen worte herzuleiten' sei (a.a. o. s.415), sucht er eine syntaktische verbindung "wo ein durch die transitivität eines anderen wortes bedingtes persönliches accusativobject auf das verbum sprechen bezogen werden kann' (ebda.). Da wendungen wie einen zu sprechen wïnschen, kriegen, bekonmen wahrscheinlich nicht so alt seien als die construction einen sprechen selbst, sei nach einer anderen verbindung umschau zu halten, und er findet diese an der Tatianstelle 59, 1: senu sín muoter inti sine bruoder stuontun úze, suohtun inan zi gisprehhanne (lat. ecce mater eius et fratres stabant foris quaerentes loqui ei). Der accusativ inan könne ebensogut vom verbum suohhen als von gisprehhan abhängig sein (vgl. suohhente thith Tat. 59,2), jedenfalls aber auf beide verba bezogen werden, während die abweichung von der lateinischen vorlage zeige, daß die wendung 'jmden zu sprechen suchen' damals eine geläufige gewesen sei. Auf welcher stufe die verschiebung zu der zeit gestanden habe, lasse sich nicht entscheiden, aber von haus aus habe jedenfalls das object zu suohhen gehört, als weitere bestimmung habe sich sodann das supinum angeschlossen, um den zweck des suchens zu bezeichnen. Die 'ähnliche syntaktische verbindung (von suohhen) auch mit anderen verben, die eine weitere verschiebung der syntaktischen gliederung gestatten' (wie Herodis suochit then kneht zi forlioscnne 'Tat. 9, 2) habe die entwicklung gefördert und zu wege gebracht, wie Wellander s. $417 \mathrm{ff}$. näher ausführt und durch weitere parallelen $\mathrm{zu}$ stützen versucht.

Hier scheint mir nun erstens die bemerkung am platze daß die abweichung von der lateinischen vorlage (ahd. inan, lat. ei) ebenso erklärlich ist, wenn es im althochdeutschen in der bedeutung. 'einen sprechen' nur ein gisprehhan mit dem accusativ, kein gisprehhan mit dem dativ, gab, und zweitens diese daß der vorausgesetzte ursprung der verbindung cinan $z i$ 
gisprehhanne suohhcn u. ä. auf schwachen füßen steht. Nelımen wir einmal an daß die verbindung von suchen mit dem supinum und einem accusativobject sich aus dem hochdeutschen oder germanischen heraus gebildet hatte, weshalb soll sie da in solcher weise entstanden sein, daß das object ursprünglich zu suchen gehörte? Weshalb soll sie einen anderen ursprung haben als die verbindungen des supinums mit verben der bedeutungen 'begehren', 'versuchen', 'denken' u. ä., bei denen doch sicher kein solcher entwicklungsgang vorliegt? Ist die uns hier vorgeführte entstehung nicht in hohem grade unwahrscheinlich? Jedenfalls bleibt uns Wellander den nachweis, sie sei wahrscheinlich, schuldig. In sätzen wie dem oben erwähnten $H$. suochit then kneht zi forliosenne, lat. $H$. quoerat puerum ad perdendum eum nimmt er eine construction $\alpha \pi \dot{o}$

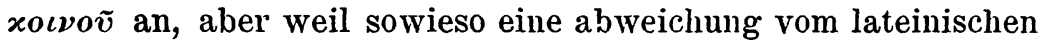
text vorliegt, kommt man mit der alleinigen beziehung von then kneht auf zi forliosenne ebenso gut aus (vgl. auch die genau entsprechende stelle im Wsä. Ev. Mtth. 2, 13 herodes secð-poet cild to forspillenne). Übrigens scheint aus dieser letzteren Tatianstelle hervorzugehen, daß die verbindung suchen mit dem supinum, wenn sie ahd. auch nur in diesem texte und bloß als wiedergabe des lat. quaero c. inf. (selber einer nachahmung des griechischen) begegnet, dem übersetzer gar nicht auffällig war, was für ihr vorheriges vorhandensein in der sprache spricht. In fällen wie suohtun inan Iudei zi arslahannc Tat. 88, 6 (ähnlich 101,2; 104, 7; 131, 15 f.; dazu noch mit inan $z i$ (ci) gifahanne 104,9; 134,10; mit inan $z i$ fahenne 124, 6; mii inan zi furliosenne, lat. quaerebant illum perdere 129,1; mit thih ci steinonne 135, 4; mit then heilant $z i$ gisehanne 114, 1; beachte die wortfolge in suohta zi gischanne inan, lat. quarebat videre cum 79,12 ) oder wie suohta Pilatus inan zi forlazzanne 198,1, suohtun inan in zi traganne 54, 2 braucht man gar nicht mit Wellander eine weiterentwicklung zu sehen, ebensowenig in suohtun in zi gangemne 113,1 oder suochit her in offane uucsan 104,1, dem einzigen falle mit. bloßem infinitiv.

Kein einziger der ahd. belege fiir suchen mit dem supinum und einem accusativ erlanbt eine entscheidung ïber die anffassung des übersetzers, indem alle betreffenden supina (m. e. 
mit einschluß von $z i$ gisprchluannc, worüber s. nachher) zu transitiven verben gehören; ander's jedoch in der gotischen

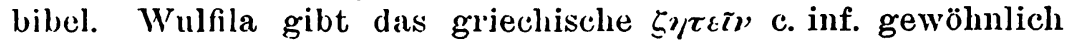
mit derselben construction (d.h. natülich hier mit dem bloßen intinitiv) wieder, vgl. Mk. 12, 12; Luk. 9, 9; 19, 3. 47; 20, 19; Joh. 7, 1. 4. 19. 20. $30 ; 8,40 ; 11,8 ; 19,12$; nur Luk. 5, 18 (= 'Tat. 54, 2) lhat er solidedun haiwa ina innatbereina für

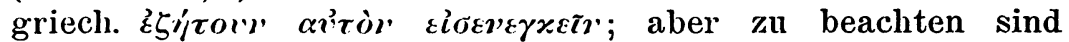
folgende fälle: jah alla managei sokidedun attekan imna,

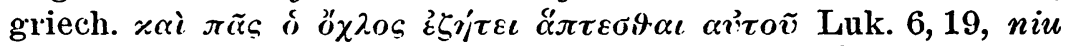

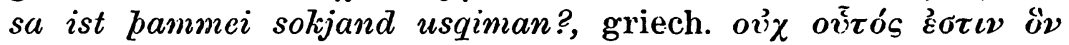

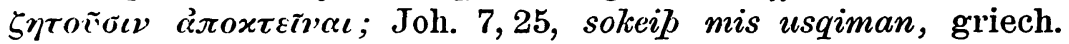

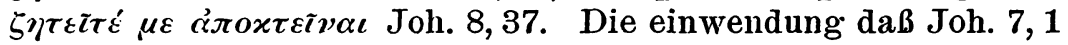

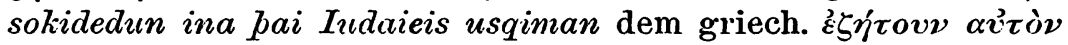

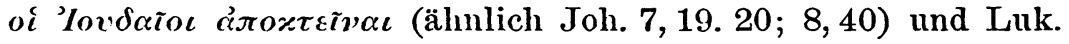

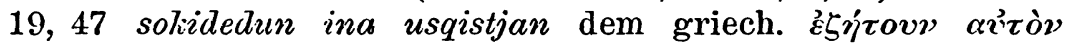

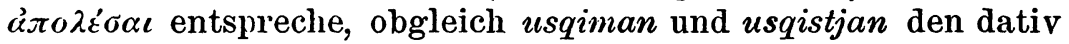
zu haben pflegen, wird durch den hinweis daß usqiman auch Mk. 12,5 (und Röm. 7,11?) und usqistjan auch Mk. 12,9 (und 3,4 ?) mit dem accusativ construiert werden, genügend entkräftet; vgl. auch fraqistjan, gewöhnlich mit dativ, aber 2 mal mit accusativ. Aus den drei obigen stellen geht hervor, daß der gotische übersetzer, m. e. auch historisch richtig, den casus als zum infinitiv gehörig empfunden hat.

Die belege im altenglischen tragen zur entscheidung dieser frage nichts bei. Von interlinearglossierungen wie das Lindisfarner Evangeliar abgesehen, finden sich 12 belege mit dem supinum, z. b. hiz hine sohton to nimanne Wsä. Ev. Jol. 7, 30; 2 olne tō: sécende sprecan (so A.; texths. fälschlich spacon) to him Mtth. 12,46 = Tat. 59,1, бa sohton para sacerda ealdras \& ba boceras hyra handa on pare tide on hine wurpan (so A.; texths. wurpun) Luk. 20, 19. Die belege, welche sämtlich in der biblischen übersetzungsliteratur und als wiedergabe des lat. quaerere c. inf. vorkommen, sind zusammengestellt́ bei Callaway, The Infinitive in Anglo-Saxon s. 286, dazu noch Mtth. 2, 13 (s. oben s. 503) ${ }^{1}$ ). Mittelenglisch war die con-

1) Von den angeblichen fünf belegen mit dem 'uninflected infinitive' bei Callaway a. a. o. sind drei zu streiclien. Blickl. Hom. 167, 2 he $\mathrm{cer}$ 
struction ziemlich beliebt (belege N.E. D. unter scek v., 11), aus dem mhd. gibt Lexer nur die eine belegstelle $\mathrm{H}$. Elisabeth, hrg. Rieger, 5883, Mhd. wb. gar keine.

Die schicksale der verbindung weiter zu verfolgen liegt außerhalb unseres gegenstandes, aber aus dem vorhergehenden dïrte klar sein daß die annahme Wellanders, inan an der Tatianstelle 59, 1 sei dem verbun suohhen zu verdanken, in der überlieferung keine stütze findet und durch einige gotischen belege vielmehr widersprochen wird. Die verbindungen welche in dem aufsatz s. $422 \mathrm{ff}$. als parallelen angeführt werden, nl. bekommen, kricgen, haben u. ä. mit dem supinum, sind anderer art und haben sich ander's entwickelt. Im niederländischen unterscheiden sie sich teilweise auch formell von der verbindung suchen mit dem supinum, welche sich solchen verben wie begchren, wünschen, probieren, wissen gleich verhält, z. b. ik heb het zoeken te verhinderen, hij heeft mij zoeken te bedriegen, dagegen $i k$ heb wat te eten gekvegen, hij heeft niets te drinken gehad, hij heeft vroeger veel te zeggen gehad, it heb me niet te belilagen gehad, nicht ${ }^{*} i k$ heb wat krijgen te eten u.s. w. $\left.{ }^{1}\right)$

Es kommt ein anderer grumd hinzu, welcher Wellanders hypothese unglaublich macht. Es wird hier der ursprung der construction sprechen mit accusativobject in einer ganz vereinzelten, vielleicht zufälligen, jedenfalls durch den wortlaut der lateinischen vorlage nahegelegten verbindung gesucht, einer verbindung, deren damalige oder spätere häufigkeit keineswegs

bone fepan so[hte] 'er suchte vorher den kampf' ist die auffassung von fépan (mit vorhergehendem artikel!) als infinitiv ausgeschlossen; on fectan 'im kampfe' begegnet auch Juliana 389 (schon Grein 'pugna?'), älnnlich noch on binum fepan Blickl. Hom. 225, 34 (Morris im Gloss. 'warfare'). Waldere A 18 und 20 feohtan sohtest 'du suchtest den kampf' ist foohtan als acc. sg. des fem. feohte die natiurlichere auffassung, und sogar wenn fcoltan eine infinitivforn wäre, so wäre dieser infinitiv als reines substantiv zu fassen und mit '(leu) kampf' zu iibersetzen, gerade wie suchte niwan strîten (mit dem subject der recke lobesam oder sim cllonthaftiu hant) Nibelungen $C^{*}$ (Bartsch) 43, $6=-$ Zarncke 7,6 (diesen nachweis rerdanke ich coll. Sijınons). Was sollte 'zu kämpfen suchen' heiben? s. nuch s. boli.

1) Unter den sonst angefiihrten parallelen hat hoi wissen mit dem supinum der objektsacensativ ganz sicher nie zu wissen gehïrt, wilhrenl verba mit der bedeutung 'anfangen, anheben' (an erster stelle begmur'n) sich gar nicht mit suchen vergleichen lasken. 
feststeht. Daß einen zu sprechen suchen je ein gewöhnlicher ausdruck gewesen sei, ist schon darum zweifelhaft, weil bei suchen doch wohl nur resultative infinitive am platze sind: man sucht etwas zu erreichen, ein ergebnis, nicht etwas zu verrichten, eine handlung. So ist es vom jetzigen niederländischen sprachgefühl aus betrachtet, so wird es aber auch überhaupt gewesen sein, und wenn ich nicht irre, steht es mit frz. chercher $\grave{a}(d e)$ nicht anders. Aus diesem grunde ist auch eine verbindung wie suochit hor in offane unesan Tat. 104, 1 wohl nur eine sklavische übersetzung und war kaum idiomatisch.

Kehren wir also zur natürlichen auffassung dab gisprehhan mit persönlichem object gutes hochdeutsch gewesen sei zurück, und versuchen wir nochmals diese construction aus sich heraus zu erklären, aber mustern wir zuerst die belege.

Den zweiten ahd. beleg gibt Wellander: mit drostu ouh thie gispreche thie sizzent innan beche Otfr. 1,10, 25, wo man m. e. mit der von Piper angenommenen bedeutung 'anreden' gut auskommt.

Im mittelniederländischen wird die beurteilung der einschlägigen fälle durch den häufigen zusammenfall der dativund accusativformen erschwert: nur wenige belege unter enen gespreken und enen spreken $\mathrm{Mnl}$. Wdb. 2, $1683 \mathrm{f}$. und 7, $1813 \mathrm{f}$. geliören sicher hierher. Einwandfrei sind aber folgende: unter enen gespreken 'einen sprechen oder anreden', 'mit einem reden': hoe selewi .. daer toe comen .. dat wine cen luttellijn ghesprelien? Parthenopeus 4948, dazu 4 belege mit unentschiedenem casus (d. h. accusativ oder dativ), sowie 3 für formell ebenfalls unsicheres hen laten gespreken 'sich überreden lassen'; - unter cnen spreken 'mit einem sprechen, eine unterredung mit einem haben': dat ic den genen wille hebben lief dic ic noit en sach meer dan heden, noch en sprac in eneger steden Ferguut 1368, Achilles ... beghcerde scer mettien, die schone Pollexina te sien ende te spreken mit goeder stade Minnen Loep 1, 3061, dazu 10 belege mit unentschiedenem casus; 'mit einem eine abmachung treffen', 'einen herbescheiden': hi sprac gocde maroniere, diene souden voeren sciere al die zee hemelike Lorreinen 2, 2097; - endlich mit dem part. prät. gesproken, welches $\mathrm{zu}^{\mathrm{u}}$ beiden verben gehört, 
'mit.. gesprochen': hi hadden te meneghen male beide ghesproken ende ghesien Couchi 1, 749, dazu 2 bèlege mit unentschiedenem casus; 'zugeredet', '(mit einem trinkgeld oder älnnlichem) gïnstig gestimmt': van den sluyswachters, dic ... niet scutten cn willen noch die schepcn dar dor laten, off sie willen gesprolien vesen Hanserecesse $8\left(10\right.$ ?), $\left.177^{1}\right)$. Zweifelhaft ist der casus in dat ghi hadt . . u vrienden (var. vriende) gesproken ende $\imath$ maghe ..., tot enen nameliken dage, van dat hy u leide te voren Grimb. Oorl. 1, 2191; u vriende $(n)$. . ende $\imath$ maghe ist der form nach accusativ, aber nach dem lochdeutschen einem von, übcr etwas sprechen erwartet man eher den dativ. Jedenfalls gehört jedoch die stelle der bedeutung wegen nicht hierher. Ein dativus pluralis findet sich in der nur im Ferguut belegten redensart den riesen gesproken hebben 'töricht handeln, seines verstandes beraubt sein': wel hebdi gesprolien den riescn Ferg. 4506, wel haddic gesproken den riesen ebda. 5154 (beide male im reim), falls Verdam (in seiner ausgabe) mit der erklärung 'eig. een gesprek met een gek (1. gekken) houden, bij een gek (1. gekken) om raad gaan' das richtige trifft ${ }^{2}$ ). Mit $\mathrm{Mnl}$. Wdb. 7, 1814 den riesen für einen acc. sing. zu halten geht m. e. nicht an, vgl. acc. sing. den ries, menighen ries bei Maerlant (Mnl. Wdb. unter ries subst.), sulken rics (v. Helten, Mnl. Spraakk. 428), dat. sing. vanden ricsc (ebda.), metten riese Spiegh. Historiael, aber der fall bleibt zweifelhaft.

In der heutigen nl. sprache, in der bekanntlich dativ und accusativ formell ganz zusammengefallen sind und gespreken durch das simplex ersetzt worden ist, ist iemand sprcken in der bedeutung 'eine (gewöhnl. kurze) unterredung mit einem haben' oder einfach 'mit einem reden' sehr gewöhnlich, z. b. $i k$ heb heni nooit gesproken 'ich bin mit ihm nie zusammengekommen', gisteren heb it hem nog gesproken 'gestern sprach ich noch mit ihm', it heb zijn vader zelf gesproken, en die heeft het mij verteld 'ich begegnete seinem vater selbst, der

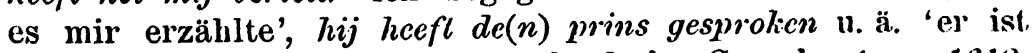
angeheitert' (vgl. Stoett, Nederlandsche Spreekw.' no. 1612,

1) Nach Verdam; ich kann die stelle nicht, finden, weil die numeriormur verschieden ist. sic und vesen weisen nuf üstliche herkunft des betrefienden textes bin.

2) In afrz. lergus fehlt cinc entsprechung. 
s. $191 \mathrm{f}$.); mit dem im neuhochdeutschen üblichen nebengedanken 'behufs besprechung einer bestimmten angelegenheit': ik zou hem graag willen spreken 'ich wïrde ihn gerne sprechen', hij moct de(n) baas sprecen, u. s. w. Daß hier alter accusativ vorliegt, ergibt sich nicht nur aus dem hochdeutschen und mittelniederländischen, sondern auch aus fällen wie is hij te spreken? 'ist er zu sprechen?', hij is vandaag slecht te spreken 'er ist heute bei übler laune' u. ä.

An letztere belege schließt sich ein neuwestfriesischer an: de baes is min to sprekken 'der meister ist ungehalten, man kann kein vernünftiges wort mit ihm reden' (Friesch Wdb. 3, 183), ferner: hy het it net fen hearren en sizzen, hy het de baes sels spritsen ' $\mathrm{er}$ hat es nicht vom sagen hören, er hat den meister selber gesprochen', d. h. 'er ist betrunken' (Stoett a.a.o. nach Waling Dijkstra, Uit Frieslands Volksleven enz. 2, 404 a).

Die beiden mnd. belege (spreken) sind schon erwähnt worden, zu den mhd. (mit gesprechen oder sprechen) s. die Wbb. und Wellander.

Ganz klar liegen die verhältnisse im altenglischen. Eine bedeutung ist 'einen anreden': AEfter Jysum zeswutelode se almihtiza zod sumum arwurðan masse-preoste be dam halzan Benedicte, and se preost ba hine zesohte on easter-tide mid lácum, swa swa him beboden was. He da hine zemette on Jam halzan easter-daze on anum scrafe and hine zesprac, and he weard pa cuð hyrdemannum, and his nama zeond eall spranz Alfric, Homil. (Thorpe) 2, 156, 16; Đa Jry cyminzas Ja hafdon lánzsume spráce wið pone zedrchtan Iob, and zewendon him haim syppan. Ac zod hi zesprac ba, and cwaed poet hé him eallum Jrim zraim ware, forpan be hi swa rihtlice atforan him ne spracon swa Iob his Jezen. Zod cwad him to: 'Nimad eow nu seofon fearras and seofon rammas, etc. ebda. 456, 26 (vgl. Hiob 42, 7 f.). Daneben findet sich häufiger die bedeutung 'mit einem eine unterredung haben', 'mit einem sprechen': $\not b a$ he drihten wolde his folc zesprecende beon in Sinai dúne Beda (Miller) 84, $4=$ (Schipper) 1, 2034 (lat. in Sina monte dominus ad populum locuturus) ${ }^{1)}$, vgl. das gleich folgende:

1) So oder ähnlich in allen hss. außer Ca.: pat da ba drihten zoolde his folc zesprecan habban, also immerhin mit dem accusativ. 
Ono nu in pare stówe bar he drihten was burh pa underJeoddan zesceafte to monnum spreocende, etc. (lat. et si illic ubi dominus per creaturam subditam hominibus loquebatur, etc.); Mid dy be heo Ja to bure cwene cóm \& heo hi zesprac \& Ja betweox him hi sp[r]accon be Oswalde, etc. ebda. hs. B (Schipper) 3, 1147, (Miller) 184, 13 (andere hss. cwom \& heo zesprecen hafdon \& betweoh oder sprcecon heo be Oswalde u. ä.; lat. cum ergo ueniens illo loqueretur cum regina, etc.) ${ }^{1)}$; Plato ... hwefde hine zesprecen Alfric, Vet. Test. (Grein) 9, 33; オa waes ic zesprecende done man Shrine 36, 19 (Bosw.-Toller). Abseits liegt der reflexive gebrauch in feower $p a$ strenzstan Jeoda hi him betweonum zesprrecon ('verabredeten sich', 'kamen überein', lat. uno agmine conspirantes) ... pot hie wolden on Romane winnan Oros. 138, 3, vgl. mhd. sich gesprechen, mnd. site spreken 'sich besprechen'. Ae. sprecan wird niemals mit dem accusativ der person verbunden.

Mittelenglisch ist ispeken überhaupt selten, dafür findet sich aber das auch ae. zesprecan vertretende speken mit persönlichem object. Zwar läßt sich hier ebensowenig wie im niederländischen streng beweisen daß früherer accusativ, nicht. dativ vorliegt, aber da die bedeutung 'mit einem sprechen', 'sich mit einem unterhalten' nur zu ae. zesprecan c. acc. pers. stimmt und ae. (ze)sprecan c. dat. pers. einen gänzlich verschiedenen sinn hatte $^{2}$ ), dürfen wir doch wohl die beiden

1) Diesen beleg verdanke ich Hesse, Perfektive und imperfektive Aktionsart im Ae. (Münster i. W. 1906) s. 88, den vorhergehenden W'ülfing 1, 216.

$\left.{ }^{2}\right)$ Der dat. pers. bei ae. (ze)sprecan fand anscheinend ziemlich beschränkte verwendung und zwar hauptsächlich mit begleitendem acc. rei, in fällen wie: poet me hearmes swa fela Adam zesproec Gen. (B) 580, to alcetanne paes fela he me laðes sproc ebda. 622, zif bu him heodas wouht hearmes zesproce ebda. 661, spracon him edwit Crist 1121, ne him swaslic word, frofre zesprcecon ebda. 1512, onzan ba pam halzan hospword sprccan Andreas 1315, ähnlich noch Ps. 108, 20, 3, Räts. 21, 33, Blickl. Hom. 99, 26, auch mit adverb: spracon me wraðc Ps. 68, 12,3; ähnlich im Heliand c. acc. rei : thie hier mid is uuordon gisprikit unveht odron (odrum M.) 1694, thuo sprak eft unaldand Crist thera idis andunivdi (theru idis and-

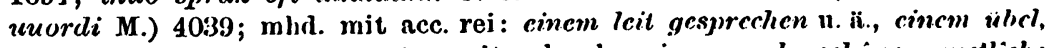

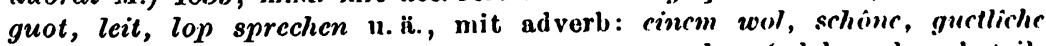
sprechen u. if.; mnl. enen lof, lachter, guact spreken (mhd. minl inul. teilweise mit der bedeutung 'cinem nachsngen' u. w., wh die Wbo.). Werner 
mittelenglischen velege welche das N.E. D. unter speak V, 32 gibt, für unsere construction in anspruch nehmen: סo cam ietro to moysen, to speken him and do kinnes-men Gen. Exod. 3400, longe haue I desired zow to speken \& se Lovelich, Grail 54, 28, um so eher als altfrz. parler a nur einheimisches speken tō (ae. sprecan tō) fördern konnte.

Aus denselben gründen sind die neuenglischen belege im N.E.D. a. a. o. aus der altenglischen construction mit dem accusativ der person herzuleiten, und zwar nicht nur diejenigen mit den jetzt veralteten bedeutungen 'einen anreden', 'einem zureden', 'sich mit einem unterhalten' (letzter beleg i. j. 1852), sondern auch die jüngeren mit der verengerten anwendung auf schiffe: to speak a ship 'ein schiff ansprechen', 'mit einem (begegnenden) schiffe sprechen' $u$. ä. (belege vom j. 1792 an bis in die jetztzeit).

Aus unseren zusammenstellungen geht nun hervor, daß an den beiden althochdeutschen stellen und an sämtlichen sechs

begegnet altenglisch der dativ in fällen in denen er nicht direct zum verbum gehört: swa wit him bu tu an sped sprecað Gen. (B) 575, hie zod herizad .. \& ealles him be naman zehwam on neod sprecað Daniel 424, vgl. mhd. einem an sîn ềe, an den eit sprechen $u$. ä. Nur einmal finde ich altenglisch mit bloßem dativ ohne ergänzung: seo tid cymo, banne ic eow ne sprece ('nicht $\mathrm{zu}$ euch sprechen werde') on biz-spellum Joh. 16, 25 (lat. cum iam non in prouerbiis loquar uobis); etwas häufiger im altsächsischen: thann sprikit im eft uualdand god Hel. 4408 (auch M. hat im, 8. Gallée, Altsächs. gramm. ${ }^{2} \S 364$, anm. 1, und der zusammenhang verlangt ' $z u$ ihnen', so daß im nicht, wie vielleicht vv. 1067. 3262, reflexiver dativ sein kann), all so he im er selbo gisprac 4932 (auch M. im 'zu ihnen', welches wieder unentbehrlich; dagegen reflexiv wohl v. 2658 gispracun im gimedlic uuord C., sprakun im M. und sicher v. 5020). Völlig wertlos sind die 14 anscheinenden belege für spreca c. dat.pers. im Lindisf. Ev. (bez. Rushw. ${ }^{2}$ ), darunter auch der N.E. D. a. a. o. angeführte aus Mtth. 12, 46 (lat. loqui ei). Ebenso ist den Tatianbelegen mit einigem miBtrauen zu begegnen, und wenn schon sprah her in uuort 74,2, man ther iu uuar sprah 131,16 idiomatisch sein können, so gilt, dieses kaum von ni sprah her in 74,2 (ähnlich 74,4), thaz Moysese spráh got 132,17 , ih sprih iu inti ir ni giloubet 134, 3, und namentlich nicht von mir ni sprihhis? 197,8. Im mittelhochdeutschen sind solche constructionen wohl gar nicht anzutreffen. - Wie dem aber auch sei, jedenfalls sind weder altenglisch, noch altsächsisch, noch althochdeutsch sprechen oder gesprechen c. dat. in der bedeutung 'cinen aureden' oder 'mit einem eine unterredung haben' belegt. 
altenglischen das compositum mit gi-, bez. $z e$ - steht. Auch mhd. ist gesprechen in dieser verwendung häufig: sofern bekannt 16 mal gegen 19 mal sprechen (vom part. prät. gesprochen abgesehen), und im mnl. ist gespreken ebenfalls nicht selten. Die bedeutung in den älteren belegen ist entweder 'einen anreden' (Otfr., AElfric 2 mal) oder 'mit einem eine unterredung haben' ('lat., Beda, Elfric 1 mal, Shrine); daß die frühere bedeutung 'einen besuchen, einen aufsuchen um mit ihm zu reden' gewesen sei, wie W. annimmt, läßt sich nicht nachweisen, würde aber für den ursprung der construction nichts verschlagen. Ein transitives gesprechen etwa im sinne 'einen mit einem gespräch treffen', 'einen zum gespräch treffen' oder 'mit einem sprechen', 'einen be-sprechen' sozusagen, ist sehr gut denkbar und es findet sich z. b. ein ähnlicher transitive gebrauch beim lat. colloqui aliquem 'einen anreden' bei Plautus (neben cum aliquo), s. Thesaurus Ling. Lat. 3, 1653 f., und beim lat. conuenire aliquem 'einen anreden, sprechen, aufsuchen', welches genau dieselben bedeutungen wie mhd. gesprechen hat.

Nachdem es einmal bei gesprechen eine construction mit persönlichem accusativ gab, konnte deren übertragung auf sprechen nicht ausbleiben. Im englischen und niederdeutschen, wo das simplex das compositum gänzlich verdrängte, war eine solche übertragung selbstverständlich, aber auch im hochdeutschen und niederländischen war sie sehr einfach. Wer die ahd. und alts. belege von gesprechen und sprechen durchmustert, ersieht bald, ein wie geringfügiger unterschied in den meisten fällen zwischen den beiden verben war. Die ausführliche erörterung der Heliand- und Genesisstellen bei H. A. J. van Swaay, Het Prefix ga- gi- ge- en de 'Actionsart' s. 240 ff. zeigt uns klar daß, von der auch altenglisch belegten bedeutung 'übereinkommen', 'verabreden' abgesehen, das compositum im altsächsischen nur selten perfective (resultative) actionsart hatte; bisweilen wurde es durativ und blob entweder dem metrum zuliebe oder zur andeutung der zeitstufe. verwendet, in anderen fällen war es linear-perfectiv ('aussprechen'), wieder teilweise mit, iibergang in die wiedergabe der zeitstufe; eine scharfe folgerichtige trennung zwischen sprekan und gisprelian war gar nicht vorhanden. Ähulich liegen die verhaitnisse bei Otfrid (und in den altenglischen 
belegen) '). Altch die sonstigen constructionen waren bei beiden verben im allgemeinen die nämlichen. Bei dieser sachlage kann die übertragung der von haus aus nur gesprechen zukommenden verbindung mit dem accusativ der person in dem sinne 'einen aureden', 'mit einem eine unterredung haben' nicht wundernehmen, und in dieser weise scheint sich mir eine ungezwungene erklärung der auffälligen verwendung des verbums sprechen im hoch- und niederdeutschen, im niederländischen, englischen und friesischen zu erbieten.
GRONINGEN, im november 1915.
J. H. KERN.

\section{DER KUCKUCK ALS ANGANGSTIER.}

Jacob Fellmann (Anteckningar under min vistelse i Lappmarken bd. 2 , s. 118. Helsingfors 1906) berichtet folgendes als einen lappischen aberglauben: 'Den som på morgunstunden på fastande mage hörde göken, var uniler hela ăret icke älskad af sin nïsta'. Als abwehrmittel gegen diese wirkung wird angegeben, man solle dreimal um eine kiefer gehen und etwas von ihrer rinde essen.

Bekanntlich ist die heidnische religion und der volksglaube der Lappen durchsetzt mit entlehnten germanischen bestandteilen. Und wemn der hier angefuihrte aberglaube auch kein besonderes merkmal frender herkunft an sich trïgt, so wird man aus seiner zusammenstellung mit den bekannten versen Walthers (Paul 48,9.10) doch ohne weiteres schließen dürfen, daß er auch den germanischen völkern geläufig gewesen ist.

Braunes deutung dieser verse (Beitr. 40, $345 \mathrm{ff}$.) kann meiner ansicht nach dadurch nur gewinnen, wenn man nummehr in ihnen nicht nur eine anspielung auf den vorstellungskreis des angangsglaubens ganz im allgemeinen, sondern einen hinweis auf einen ganz bestimmten damals noch lebendigen aberglauben sehen darf. ${ }^{2}$ )

MARBURG.

WOLF VON UNWER'TH.

1) Im Beowulf ist nach A. Lorz, Actionsarten des verbums im Beowulf, Würzburger diss. 1908 , s. 78 f. das compositum manchmal nachdriicklicher als das simplex, eine sehr subjective anschauung.

2) [Bei dankbarer anerkennung des obigen nachweises kann ich trotzdem nicht zugeben, daß derselbe für Deutschland etwas bedeute. Es bleibt dabei, daß in den reichen mhd. und nhd. belegen weder esel noch kuckuck als deutsche angangstiere bezengt sind. Ich $\mathrm{muB}$ deshalb daran festhalten, daß Walther die wirklich bekannten angangstiere genannt haben würde, wenn er nicht bloß im allgemeinen nit dem angangsalverglauben hätte spielen wollen. - W. B.] 